\title{
Modeling the land-use correlates of vehicle-trip lengths for assessing the transportation impacts of land developments
}

\section{Sivaramakrishnan Srinivasan ${ }^{a}$ \\ University of Florida}

\author{
Russell Provost \\ University of Florida
}

\author{
Ruth Steiner \\ University of Florida
}

\begin{abstract}
This study developed models that relate trip lengths to the land-use characteristics at the trip ends (both production and attraction ends). Separate models were developed by trip purpose. The results indicate several statistically significant and intuitively reasonable effects of land-use patterns. High residential densities and a good mix of complementary land uses are associated with shorter trips. Larger establishments attract longer trips, and the lengths of home-based other trips decrease with an increase in the number of convenient commercial landuse parcels in the neighborhood. The connectivity provided by the roadway network and the urban form of the area (measured in terms of number of intersections and cul-de-sacs) affect trip lengths. In addition to the local land-use characteristics, trip lengths also vary significantly by the location of the neighborhood within the region. All these results hold even after controlling for several trip and traveler characteristics. The models have been implemented in a spreadsheet program for use in assessing the transportation impacts of new land developments.
\end{abstract}

\section{$1 \quad$ Background}

There is continued interest in understanding and quantifying the relationships between land use/built environment and various aspects of travel behavior such as trip rates, trip-chaining patterns, mode choice, and travel distances. The focus of this study is on understanding the nexus between land-use patterns and vehicle-trip distances from the standpoint of assessing the transportation impacts of new land developments. Traditionally, transportation-impact assessment methods have relied only on trip rates as the metric of impact. While this is appropriate to capture the effect of a development locally (say, at a nearby intersection), it is not an adequate measure of system-wide impacts. For instance, two developments could result in the same number of additional trips, but one of them could be attracting these trips from much farther away. In this case, it could be argued that the transportation impacts of both these developments are not identical (as would be indicated by an assessment based purely on trip volume); rather, the one that leads to longer trip lengths effectively has a greater (negative) impact on the transportation system. With increasing emphasis on growth management and the containment of urban sprawl (in addition to congestion mitigation), there is a need for the assessment of such macro impacts of development using methods that relate the built-environment patterns to trip lengths.

In addition, there is a desire to moderate or reduce the energy consumed by the transportation sector to achieve energy sustainability and to reduce the extent of greenhouse gas (GHG) emissions from vehicles. To achieve this goal without adversely affecting the quality-of-life of people (broadly defined as the ability of people to satisfy their activity-participation needs), planners and policymakers are exploring urban-design solutions, such as mixed-use neighborhoods. To assess the extent to which such land-use patterns can reduce the length of travel undertaken, it is necessary to quantify the relationships between land use and trip lengths.

A brief overview of the literature on the relationships between land use and trip lengths is presented here. (For a more-detailed discussion of the literature on the relationships between land use and other aspects of travel behavior, such as trip rates and mode choice, see Ewing and Cervero 2001; and TRB 2009.) Most studies examine total household (or person-level) vehicle miles traveled (VMT) as a function of the land-use patterns around the residence of the household. Early works of Handy (1996), Ewing et al. (1994), and Rutherford (1996) suggest traditional neighborhood developments (TND) produce shorter trips when compared to conventional suburban subdivisions. Cervero and Kockelman (1997) report that VMT by personal automobiles is less in households located in areas with higher accessibility. Ewing et al. (1994) compared the vehicle hours of travel (VHT) for six neighborhoods and found that the compact neighborhoods with higher

a siva@ce.ufl.edu 
densities and a greater mix of uses generated lower levels of VHT. Brownstone and Golob (2009) report that residences in higher-density neighborhoods (the only land-use variable used in their models) have lower VMT. Holtzclaw et al. (2002) also report a similar effect of density. Further, they also find that bicycle and pedestrian friendliness are less-strongly related to household VMT. Cervero and Duncan (2006) examined the VMT associated with work and shopping travel individually. Their analysis indicates that both jobs-housing balancing and retail-housing balancing are good strategies for reducing VMT, with the former being more effective than the latter. Bagley and Mokhtarian (2002), however, find that residential location type (extent to which the neighborhood is "traditional") has little impact on trip lengths after controlling for attitudinal and lifestyle factors and using cross-sectional data. Handy et al. (2005) also concur with this assessment. However, they also report that with the use of quasi-longitudinal data, the builtenvironment characteristics are statistical predictors for changes in volume of driving. For instance, they find that increased accessibility is associated with decreased driving. Based on another longitudinal study, Krizek (2003) also reports a decrease in household VMT with an increase in neighborhood accessibility. Unlike the previous studies, which have measured the land use around the residence locally, Bento et al. (2005) relate the household VMT to the characteristics of the urban area in which the household resides and find that VMT increases with greater sprawl.

The objective of our study is to contribute to the knowledge on the empirical relationship between land use and trip lengths. Unlike the previous studies on household VMT, we focus on the lengths of individual trips. Therefore, we capture the effects of land use at the trip ends instead of only residential-location characteristics. The study undertaken by Frank et al. (2005) also involves constructing land-use variables at all trip ends based on a buffer around the trip-end location. However, their models for household VMT still appear to consider only the land use around the residential location.

Our approach is appropriate for calculating the trip lengths associated with different types of land developments (transportation-impact analysis). It is anticipated that in a traffic-impact study, only the land-use data around the land parcel being developed will be available, and we therefore use land-use descriptors only at one end of the trip (the end where developments are being proposed). Thus, this study adopts a "development-centric" approach.

Further, we describe trip-end land use at three different spatial levels: (1) the characteristics (e.g., building square footage in the parcel) of the land parcel in which the trip ends, (2) the characteristics (e.g., percentage of residential and commercial square footage) of the "neighborhood" in which the parcel is located, and (3) the characteristics of the location of the neighborhood within the region (e.g., distance to regional activity centers).

The rest of this paper is organized as follows. The data used in the analysis are described in the next section. The empirical modeling results are presented and discussed in Section 3. An application of the estimated models for predicting trip lengths is discussed in Section 4. Section 5 concludes this paper and identifies directions for future research.

\section{Data}

The intent of this study is to develop models to relate trip lengths as a function of land-use and network characteristics. The models also control for the socioeconomic characteristics of the traveler, which could influence trip lengths. Thus, data on travel patterns, land-use characteristics, roadway network, and traveler characteristics are needed. The rest of this section describes the sources of these data and the procedure employed to assemble the data for model estimations.

The 1999 Southeast Florida Regional Travel Characteristics Study is the primary source of data for this analysis. In this survey, one-day travel information was collected from about 5000 households in Miami-Dade, Broward, and Palm Beach Counties. The following information was collected for each trip undertaken by each respondent: trip timing (start and end times), mode (including occupancy for auto mode), purpose, and trip-end locations (addresses). In addition, the researchers were also provided with the geo-coded (latitudes and longitudes) trip-end locations. The travel survey also provides information on selected socioeconomic characteristics of the traveler such as age, employment status, automobile ownership, household structure, and household income. Quite interestingly, the gender of the travelers was not obtained in the travel survey.

The roadway characteristics were obtained from the statewide roadway network file titled "Dynamap Streets," made available on the Florida Standard Urban Transportation Model Structure (FSUTMS) web portal (http://www.fsutmsonline. net/index.php?/gisonline/). The roadway data represent year 2005. As information on the year in which each roadway segment was built was unavailable, it was not possible to adjust the roadway network to reflect 1999 (survey year) conditions. The "Network Analyst" toolset within ArcGIS was used to determine the network distance of each trip (based on the shortest-distance path) in the survey (excluding those trips for which the trip-end locations were unknown or were outside the three-county region).

The discussion thus far has focused primarily on determining the "dependent" variable of interest: the trip lengths. The second component of data required is the land-use and transportation-system characteristics or the "explanatory variables" for use in the models. The primary source of this information is the Florida Department of Revenue (FDOR). These 
parcel data files contain valuable information for our analysis including the land-use type, area of buildings, and the number of residential units located on each parcel.

The parcel data obtained reflect the land-use characteristics in the year 2008. However, the travel survey data are from 1999. To ensure consistency, developments built after 1999 were removed and subjected to significant cleaning and updating.

The cleaned parcel-level file created contains the following details for each parcel in the three-county region: (1) a parcel identifier, (2) parcel area, (3) land-use type, (4) number of residential units for residential parcels, and (5) square footage for nonresidential buildings. The original FDOR database has 99 categories for land-use type. These were aggregated to create a more manageable and practically useful land-use classification scheme that includes six categories: (1) residential (singlefamily, multi-family, and mobile homes), (2) commercial (large retail, regular retail, convenience stores, and drive-through establishments), (3) office (professional and nonprofessional services buildings), (4) industrial (light, heavy, and warehousing), (5) institutional, and (6) other.

In the next step, "neighborhoods" were created and were characterized by aggregating the data from the cleaned parcellevel files and the transportation-network files. In this study, neighborhoods are defined as grid cells four square miles in size. To generate these neighborhoods, a four-square-mile grid was arbitrarily imposed on the study region (three-county region). Each cell in this grid was assigned a unique "neighborhood identifier" value. The entire grid was then shifted horizontally by one mile. The shifted grid cells were assigned new "identifier" values. In the third step, the grid from the previous step was shifted vertically by one mile and assigned new identifier values. Effectively, this procedure creates a set of overlapping neighborhoods four square miles in size across the entire region with the centroids of these neighborhoods lying on a one-square-mile grid (Figure 1).

The characteristics of the neighborhood were determined by aggregating the parcel-level data. Specifically, each parcel was assigned to one or more neighborhoods if the parcel centroid fell within the corresponding boundaries (note that each parcel can be assigned to multiple neighborhoods as the neighborhood areas themselves overlap). Once the land parcels were mapped to the neighborhoods, the land-use descriptors of the neighborhood (such as land area under different land uses and building square footage under different uses) could be obtained by spatial aggregation. Similarly, the roadway network data were also aggregated to determine measures, such as linear road miles, number of intersections, and number of cul-de-sacs within each neighborhood. The incorporation of other descriptors of the roadway network topology (see for example, Parthasarathi and Levinson 2011; and Parthasarathi et al. 2011) is identified as an extension of this study.

\section{Neighborhood Delineation}

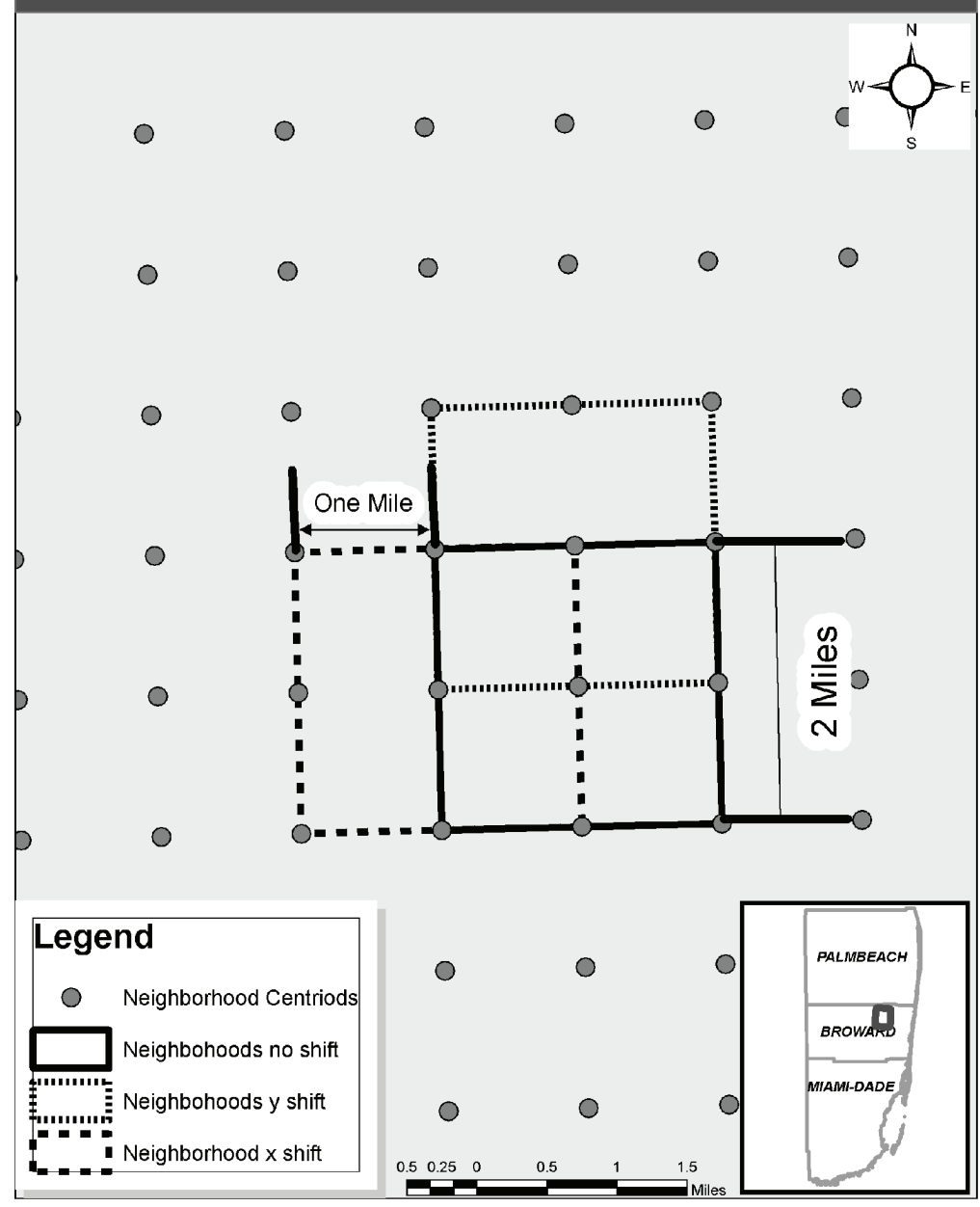

Figure 1: Neighborhood delineation.

As already mentioned, the intent of this study is to capture land use at three spatial levels: parcel, neighborhood, and regional. The development of parcel and neighborhood characteristics has been discussed thus far. Next, we present the approach used to describe the location of each neighborhood within the three-county study region. Two measures were constructed:

- The network distance of each neighborhood to each of four regional activity centers (one each in Miami, Fort Lauderdale, Boca Raton, and Palm Beach) defined as neighborhoods with the highest commercial square footage (includes, retail, office, and entertainment).

- The network distance of each neighborhood to each of nine regional residential centers. Residential centers were defined as neighborhoods having more than 50 percent of their land use dedicated to residential. Among these, three neighborhoods in each county (for a total of nine neighborhoods geographically distributed across the study region) that had the largest number of residential units were selected as the regional residential centers. 
Once all the land-use variables at the parcel, neighborhood, and regional levels were created, they were suitably linked to the trip-end locations from the household-travel survey data file. First, each trip-end location was matched to a specific land parcel based on the proximity of the trip-end location to the parcel centroid. Each trip-end location was also matched to a neighborhood based on the proximity of its location to the neighborhood centroid. As the neighborhoods are overlapping, the trip-end locations are likely to fall closer to the centroids than an edge.

After the overall data assembly process, the final database comprises more than 30,000 trips with each trip characterized by the distance and land-use characteristics (at the different spatial levels) at each trip end (these are in addition to the other attributes such as timing, mode, and purpose obtained directly from the survey). These 30,000 trips include cases with missing information on one or more attributes. A clean analysis sample was extracted that includes non-missing and acceptable values for all required attributes. As the focus is on vehicle trips, trips not made using the auto were removed; this includes the removal of trips with no known mode of travel. Similarly, those trips made by an auto but as a passenger were also not included, as the corresponding trips could also have been reported by the driver of the vehicle. After removing the trips that could not be accurately matched to parcels and neighborhoods and those trips that were unreasonable (very short or very long) in terms of trip distances, travel times, and speeds, the final analysis sample consisted of approximately 18,000 trips. Of these, 5237 were home-based work (HBW) trips; 8257 were homebased other (HBO) trips; and 4796 were non-home-based (NHB) trips. Descriptive characteristics of the trip lengths (in miles) for the three trip purposes are presented in Table 1. Summary statistics on the land-use characteristics at the production and attraction ends of the trips (across all trip purposes) are presented in Table 2. 
Table 1: Descriptive statistics for trip lengths (in miles) by purpose.

\begin{tabular}{|c|r|r|r|r|r|r|}
\hline & \multicolumn{2}{|c|}{ Home-Based Work (HBW) } & \multicolumn{2}{c|}{ Home-Based Other (HBO) } & \multicolumn{2}{c|}{ Non-Home Based (NHB) } \\
\cline { 2 - 7 } & Trip Length & LN (Trip Length) & Trip Length & LN (Trip Length) & Trip Length & LN (Trip Length) \\
\hline Number of trips & 5327 & 5327 & 8257 & 8257 & 4796 & 4796 \\
\hline Mean & 10.31 & 1.93 & 5.42 & 1.16 & 6.14 & 1.26 \\
Median & 7.70 & 2.04 & 3.30 & 1.19 & 3.92 & 1.37 \\
\hline Variance & 93.04 & 0.98 & 39.79 & 1.17 & 48.77 & 1.31 \\
\hline Minimum & 0.10 & -2.28 & 0.10 & -2.29 & 0.10 & -2.28 \\
\hline 5 Percentile & 1.09 & 0.09 & 0.49 & -0.71 & 0.43 & -0.83 \\
10 Percentile & 1.91 & 0.65 & 0.76 & -0.27 & 0.76 & -0.28 \\
90 Percentile & 21.20 & 3.05 & 12.79 & 2.55 & 14.15 & 2.65 \\
95 Percentile & 27.99 & 3.33 & 17.11 & 2.84 & 18.94 & 2.94 \\
\hline Maximum & 97.75 & 4.58 & 85.00 & 4.44 & 82.62 & 4.41 \\
\hline
\end{tabular}

Table 2: Descriptive statistics for trip-end land uses.

\begin{tabular}{|c|c|c|c|c|c|c|c|c|}
\hline & \multicolumn{4}{|c|}{ Land-Use at Production End } & \multicolumn{4}{|c|}{ Land-Use at Attraction End } \\
\hline & Mean & $\mathrm{SD}$ & $5 \%$ ile & $95 \%$ ile & Mean & SD & $5 \%$ ile & $95 \%$ ile \\
\hline \multicolumn{9}{|l|}{ Parcel Characteristics } \\
\hline \multirow{7}{*}{$\begin{array}{l}\text { Residential }(0 / 1) \\
\text { Commercial }(0 / 1) \\
\text { Office }(0 / 1) \\
\text { Institutional }(0 / 1) \\
\text { Industrial }(0 / 1) \\
\text { Other }(0 / 1) \\
\text { Building Area* }\end{array}$} & 0.716 & 0.451 & 0.000 & 1.000 & 0.266 & 0.442 & 0.000 & 1.000 \\
\hline & 0.106 & 0.308 & 0.000 & 1.000 & 0.333 & 0.471 & 0.000 & 1.000 \\
\hline & 0.055 & 0.227 & 0.000 & 1.000 & 0.154 & 0.361 & 0.000 & 1.000 \\
\hline & 0.046 & 0.210 & 0.000 & 1.000 & 0.108 & 0.311 & 0.000 & 1.000 \\
\hline & 0.024 & 0.154 & 0.000 & 1.000 & 0.062 & 0.241 & 0.000 & 1.000 \\
\hline & 0.053 & 0.224 & 0.000 & 1.000 & 0.077 & 0.267 & 0.000 & 1.000 \\
\hline & 21.069 & 97.339 & 0.000 & 111.764 & 58.581 & 159.363 & 0.000 & 271.465 \\
\hline \multicolumn{9}{|l|}{ Neighborhood Land-use Characteristics } \\
\hline \multirow{14}{*}{$\begin{array}{l}\text { Fraction of land area that is developed } \\
\text { Fraction of developed area that is residential } \\
\text { Fraction of developed area that is commercial } \\
\text { Fraction of developed area that is office space } \\
\text { Fraction of developed area that is institutional } \\
\text { Fraction of developed area that is industrial } \\
\text { Fraction of developed area that is other land use } \\
\text { Net residential density } \\
\text { Building area (Commercial) } \\
\text { Building area (Office) } \\
\text { Building area (Institutional)* } \\
\text { Building area (Industrial) } \\
\text { Building area (Other)* } \\
\text { Number of convinient-commercial parcels in neighborhood }\end{array}$} & 0.525 & 0.110 & 0.324 & 0.674 & 0.525 & 0.114 & 0.303 & 0.675 \\
\hline & 0.669 & 0.157 & 0.377 & 0.881 & 0.622 & 0.178 & 0.246 & 0.856 \\
\hline & 0.064 & 0.052 & 0.000 & 0.166 & 0.074 & 0.054 & 0.010 & 0.184 \\
\hline & 0.032 & 0.045 & 0.000 & 0.108 & 0.046 & 0.063 & 0.002 & 0.142 \\
\hline & 0.071 & 0.060 & 0.008 & 0.156 & 0.078 & 0.069 & 0.011 & 0.182 \\
\hline & 0.045 & 0.080 & 0.000 & 0.186 & 0.063 & 0.106 & 0.000 & 0.270 \\
\hline & 0.119 & 0.120 & 0.008 & 0.360 & 0.117 & 0.119 & 0.009 & 0.347 \\
\hline & 8.658 & 6.069 & 2.811 & 15.769 & 9.377 & 7.420 & 3.344 & 17.356 \\
\hline & 1077.115 & 1225.346 & 0.000 & 3127.491 & 1307.475 & 1376.576 & 69.510 & 3785.170 \\
\hline & 957.759 & 1960.591 & 0.000 & 4375.543 & 1465.733 & 2658.360 & 10.932 & 6088.734 \\
\hline & 794.822 & 997.771 & 17.506 & 2384.259 & 945.738 & 1190.166 & 36.388 & 3245.766 \\
\hline & 942.671 & 1813.638 & 0.000 & 4350.773 & 1303.696 & 2379.505 & 0.000 & 5608.807 \\
\hline & 289.371 & 459.114 & 7.047 & 1205.701 & 348.510 & 487.057 & 15.077 & 1346.287 \\
\hline & 10.290 & 9.490 & 0.000 & 29.000 & 11.420 & 9.615 & 1.000 & 32.000 \\
\hline \multicolumn{9}{|l|}{ Neighborhood Roadway Characteristics } \\
\hline \multirow{3}{*}{$\begin{array}{l}\text { Road miles } \\
\text { Intersections per mile } \\
\text { Cul-de-sacs per mile }\end{array}$} & 70.834 & 21.677 & 28.941 & 102.324 & 71.349 & 21.965 & 28.631 & 105.281 \\
\hline & 8.643 & 1.718 & 5.665 & 10.981 & 8.900 & 1.573 & 6.211 & 11.048 \\
\hline & 1.502 & 0.769 & 0.430 & 2.859 & 1.429 & 0.758 & 0.415 & 2.786 \\
\hline \multicolumn{9}{|l|}{ Location of Neighborhood within Region } \\
\hline \multirow{3}{*}{$\begin{array}{l}\text { Distance to nearest regional activity center } \\
\text { Range of distances to activity cetners } \\
\text { Distance to nearest regional residential center }\end{array}$} & 10.198 & 5.560 & 2.167 & 18.686 & 9.064 & 5.457 & 1.510 & 17.149 \\
\hline & 48.816 & 13.519 & 27.743 & 67.602 & 49.625 & 13.591 & 27.900 & 67.602 \\
\hline & 8.778 & 5.772 & 2.040 & 18.864 & 8.179 & 5.440 & 2.148 & 17.738 \\
\hline
\end{tabular}

* building area is in square feet and applicable only for non-residential land use (equals zero for residential parcels) 


\section{Empirical results}

This section of the paper presents the empirical models estimated to relate trip lengths to land-use characteristics. The logarithm of the trip length is taken as the dependent variable (this approach guarantees that the predicted trip lengths are always positive), and these are related to the land-use and traveler characteristics using a linear-regression structure. The use of advanced econometric methods is identified as an area of future enhancement.

Separate models were estimated for each trip purpose, and these are presented in Tables 3 (home-based work), 4 (homebased other), and 5 (non-home-based). For each trip purpose, two sets of models were developed. The first examines the impacts of land use at the production end of trips, and the second examines the impacts of land use at the attraction end of trips. These are labeled "production-end models" and "attraction-end models" in Tables 3, 4, and 5. Further, for each trip purpose and within each of the production-end and attraction-end model categories, two empirical specifications are presented. The first, called the "aggregate" model, includes only land-use and transportation-system characteristics as explanatory factors, and the second, called the "disaggregate" model, also includes trip and traveler characteristics as explanatory variables. Thus, a total of 12 models were estimated; these are discussed by trip purpose. Note that a hyphen ( - ) corresponding to any variable in Tables 3,4 , or 5 indicates a statistically insignificant effect. Similarly, "NA" indicates that the corresponding variable was not applicable and hence not used in the model under consideration.

\subsection{Models for the lengths of home-based work (HBW) trips}

Table 3 presents the models for the lengths of home-based work (HBW) trips. The explanatory factors are classified into the following six categories: (1) parcel characteristics, (2) neighborhood land-use characteristics, (3) neighborhood roadway characteristics, (4) location of neighborhood within region, (5) trip characteristics, and (6) traveler characteristics. The first four categories of variables refer to the land use at the home end of the trips in the "production-end" models and to the non-home end of the trips in the "attraction-end" models. The fifth and sixth categories of variables are applicable only for the disaggregate models.

The first category of explanatory variables is parcel characteristics. Each parcel is characterized by a land-use type, which can be one of the following: residential, commercial, office, institutional, industrial, or other. Home-based trips are, by definition, produced at residential parcels, and hence the land-use type variable is applicable only to the attraction-end models. HBW trips attracted to institutional parcels are of the shortest length compared to similar trips attracted to any other type of parcel. HBW trips attracted to commercial parcels are longer than trips attracted to institutional parcels, but are shorter compared to trips attracted to residential, office, and other parcels. A second parcel-level characteristic is the square footage of the building in the parcel. This attribute was also defined for only nonresidential parcels, and the attraction-end models indicate that larger-size establishments attract longer HBW trips. Residential parcels were characterized in terms of the number of units in the parcel (single unit, 2 to 10 units, and more than 10 units). No statistically significant differences were estimated in trip lengths produced across these categories. This is possibly because a very large number of residential parcels had only one residential unit.

The next category of explanatory variables is neighborhood land-use characteristics. The first variable of interest is the fraction of area that is developed, calculated as the ratio of the sum of the areas in the six land-use categories (residential, commercial, office, institutional, industrial, and other) to the total area of the neighborhood. In most cases, the total area of the neighborhood is four square miles based on how the neighborhoods were delineated. However, for neighborhoods along the coast and along the boundaries of the study region, the total area could be smaller. In all four models, the coefficient on this variable is negative, indicating that HBW trips produced in and attracted to the more-developed neighborhoods are shorter.

The next set of variables captures the fraction of developed area by each land-use type. Neighborhoods with a greater proportion of residential land use produce longer HBW trips but attract shorter HBW trips. If the neighborhood is largely residential, then there would not be as many opportunities for employment in the vicinity and consequently, the HBW trips produced would be longer. However, any employment center located in that neighborhood could likely draw its employees from the large pool of residents in its vicinity, leading to shorter trips being attracted. HBW trips produced in neighborhoods with a greater fraction of "other" area are also estimated to be longer. Alternatively, one could interpret the model to imply that a greater fraction of nonresidential, non-other land use (i.e., greater fraction of commercial, office, institutional, or industrial) would lead to shorter HBW trips being produced. Residential density (number of residential units in the neighborhood divided by the area of the neighborhood that is residential) is negatively correlated with the lengths of HBW trips. This implies that work trips produced in and attracted to parcels in high-density neighborhoods are shorter, a result that is consistent with findings in the literature. 
Table 3: Model for HBW trip lengths.

\begin{tabular}{|c|c|c|c|c|c|c|c|c|}
\hline & \multicolumn{4}{|c|}{ Production-End Models } & \multicolumn{4}{|c|}{ Attraction-End Models } \\
\hline & \multicolumn{2}{|c|}{ Aggregate } & \multicolumn{2}{|c|}{ Disaggregate } & \multicolumn{2}{|c|}{ Aggregate } & \multicolumn{2}{|c|}{ Disaggregate } \\
\hline & Param. & t. stat. & Param. & t. stat. & Param. & t. stat. & Param. & t. stat. \\
\hline \multicolumn{9}{|l|}{ Parcel Characteristics } \\
\hline Commercial land use & NA & NA & NA & NA & -.088 & -2.806 & -.077 & -2.475 \\
\hline Institutional land use & NA & NA & NA & $\mathrm{NA}$ & -.130 & -2.693 & -.133 & -2.788 \\
\hline Building area & NA & NA & NA & NA & 2.67E-04 & 3.017 & $2.49 \mathrm{E}-04$ & 2.852 \\
\hline \multicolumn{9}{|l|}{ Neighborhood Land Use Characteristics } \\
\hline Fraction of area that is developed & -.209 & -1.456 & -.170 & -1.196 & -.620 & -4.404 & -.588 & -4.015 \\
\hline Fraction of developed area that is residential & .527 & 3.983 & .513 & 3.915 & -.452 & -5.435 & -.418 & -5.100 \\
\hline Fraction of developed area that is other land use & .781 & 4.638 & .721 & 4.320 & & & & \\
\hline Net residential density (units per acre) & -.005 & -1.781 & -.006 & -1.940 & -.004 & -2.023 & -.004 & -1.796 \\
\hline LN (Building area - Commercial) & - & - & - & - & -.061 & -4.912 & -.055 & -4.504 \\
\hline LN (Building area - Office) & -0.29 & -3.313 & -.030 & -3.437 & .026 & 2.542 & .021 & 2.098 \\
\hline LN (Building area - Industrial) & - & - & - & - & .010 & 1.665 & .011 & 1.866 \\
\hline LN (Building area - Other) & - & - & - & - & .020 & 1.874 & .021 & 1.961 \\
\hline \multicolumn{9}{|l|}{ Neighborhood Roadway Characteristics } \\
\hline Intersections per mile of roadway & -.045 & -4.309 & -.033 & -3.202 & - & - & - & - \\
\hline Cul-de-sacs per mile of roadway & .039 & 2.045 & .040 & 2.106 & - & - & - & - \\
\hline \multicolumn{9}{|l|}{ Location of Neighborhood within Region } \\
\hline Distance to nearest regional activity center & .025 & 7.811 & .025 & 7.839 & -.099 & -2.608 & -.007 & -2.027 \\
\hline Range of distances to regional activity centers & - & - & - & - & .055 & 4.296 & .005 & 4.945 \\
\hline Distance to nearest regional residential center & -.018 & -6.595 & -.017 & -6.292 & -.011 & -3.704 & -.011 & -3.784 \\
\hline \multicolumn{9}{|l|}{ Trip Characteristics } \\
\hline AM peak & & & -.078 & -2.285 & & & -.062 & -1.766 \\
\hline Mid day & & & -.243 & -5.715 & & & -.249 & -5.768 \\
\hline PM peak & & & -.075 & -2.045 & & & -.061 & -1.647 \\
\hline \multicolumn{9}{|l|}{ Traveler Chracteristics } \\
\hline Full-time employed & & & .201 & 4.576 & & & .136 & 3.038 \\
\hline Age $26-35$ & & & .235 & 5.343 & & & .185 & 4.151 \\
\hline Age $36-45$ & & & .150 & 3.519 & & & .113 & 2.617 \\
\hline Age 46-55 & & & .094 & 2.165 & & & .092 & 2.092 \\
\hline Age 56-65 & & & .087 & 1.646 & & & .083 & 1.550 \\
\hline Fewer cars than adults in household & & & -.077 & -2.437 & & & -.126 & -3.946 \\
\hline Income $<=40 \mathrm{~K}$ & & & -.141 & -3.745 & & & -.205 & -5.382 \\
\hline Income $>=80 \mathrm{~K}$ & & & .102 & 3.026 & & & .146 & 4.307 \\
\hline Constant & 2.003 & 12.447 & 1.596 & 9.087 & 2.571 & 18.947 & 2.318 & 15.110 \\
\hline Adjusted R ${ }^{2}$ & \multicolumn{2}{|c|}{0.067} & \multicolumn{2}{|c|}{0.093} & \multicolumn{2}{|c|}{0.054} & \multicolumn{2}{|c|}{0.082} \\
\hline Standard deviation of error & \multicolumn{2}{|c|}{0.957} & \multicolumn{2}{|c|}{0.944} & \multicolumn{2}{|c|}{0.968} & \multicolumn{2}{|c|}{0.953} \\
\hline
\end{tabular}


In addition to the proportion of area under different landuse types, the size of the buildings (square feet) is also found to be a strong predictor of trip lengths. HBW trips produced in neighborhoods with large office spaces are shorter, whereas those attracted to neighborhoods with large office spaces are longer. HBW trips attracted to neighborhoods with large industrial and other floor space are also longer; however, such trips attracted to areas with large commercial space are shorter (relative to trips attracted to areas that have large office, industrial, or other floor spaces). If the neighborhood has a large nonresidential floor area, the trips attracted to such neighborhoods could not have been produced in the vicinity of the attraction end and hence are longer.

The third category of explanatory variables is neighborhood roadway characteristics. These are statistically significant in only the production-end (i.e., home) models. The greater the intersections per mile of roadway, the shorter the trips, and the more cul-de-sacs per mile of roadway, the longer the HBW trips. These variables are descriptive of the urban form of the neighborhood. A neighborhood characterized by cul-de-sacs represents traditional suburban-style residential development, whereas a neighborhood characterized by a grid street network represents traditional or New Urbanist development. The directionality of the impacts of the urban form of the neighborhood is intuitively reasonable.

The fourth category of explanatory variables is location of the neighborhood within the region. With increasing distance of the production end (neighborhood) of the trip to the regional activity centers, the lengths of HBW trips increase. Alternatively, HBW trips produced closer to regional activity centers are shorter, potentially due to the increased number of opportunities located near the neighborhood. At the same time, HBW trips produced near the large residential centers are longer, possibly because of the limited employment opportunities in the vicinity of the production end of the trip.

With an increasing distance of the attraction end (neighborhood) of the trip to the regional activity centers, the lengths of home-based trips decrease. Alternatively stated, regional activity centers attract the longest HBW trips. The next variable is the "range of distances to the regional activity centers." This is a measure of centrality of the attraction location relative to the regional activity centers. If the range of distances (difference in distances to the farthest and closest regional activity centers) is large, the location under consideration is significantly closer to one of the activity centers. Alternatively, if the range is small, the location is more "central" relative to the regional activity centers. The coefficient on this variable is positive, indicating that centrally located neighborhoods attract shorter HBW trips. Finally, the models also indicate that HBW trips attracted to locations near the large residential centers are longer. This could be reflective of the types of jobs in those areas-perhaps the people who work in commercial establishments in large residential centers often do not live in the same locality.

The fifth and sixth categories of variables are applicable to only the disaggregate models. Among trip characteristics, HBW trips made midday (9 a.m. to 3 p.m.) are the shortest and those made during the off-peak periods (before $7 \mathrm{a} . \mathrm{m}$. or after 6 p.m.) are the longest. The time of day of the trip was determined as the midpoint time of the trip. On examining the impacts of traveler characteristics, we find that full-time workers make longer HBW trips compared to part-time workers. The length of HBW trips decreases with increases in the age of the traveler. The gender of the traveler was not obtained in the travel survey. HBW trips made by individuals who have to share cars (fewer cars than adults in the household) are shorter. Finally, we see that the length of HBW trips increase with increases in household income. The excluded income categories in the model are "income between $\$ 40,000$ and $\$ 80,000$ " and "unknown income." Thus, we find that HBW trips made by persons who did not report their income are comparable in length to those made by persons with income between $\$ 40,000$ and $\$ 80,000$. In general, these results are intuitively reasonable. Further, the land-use variables still retain their significance even after controlling for these trip and traveler characteristics. No data on attitudes and lifestyles were collected in the travel survey.

The estimated variances of the error terms are large, or equivalently, the $\mathrm{R}^{2}$ values are small. This indicates that the included variables "explain" the variability in trip lengths only to a limited extent (between 5 percent and 9 percent in the variability in the logarithm of the trip lengths across the four models). Further, on comparing the aggregate and disaggregate models, we see that the land-use variables explain the variability to a greater extent than the socio-economic variables included in the model.

Data on the types of employment and housing costs were not available for this study. We believe that capturing the availability of housing that corresponds to the types of jobs in an area (and vice versa) would improve the predictive power of the model. Further, the length of HBW trips fundamentally depends on the choice of residential location (relative to work location), which in turn depends on factors such as the work and school location of other household members. Current models do not control for these factors either. Finally, the data on the socio-economic characteristics of the traveler are limited. In particular, the survey did not collect data on the gender of the traveler, a factor that is well known to influence the length of work trips. Thus, there are clear avenues to empirically enhance the current model subject to availability of additional data. 


\subsection{Models for the lengths of home-based other (HBO) trips}

Table 4 presents the models for the length of home-based other (HBO) trips. The structure of this table is similar to that of Table 3.

The first category of explanatory variables is parcel characteristics. Home-based trips are, by definition, produced at residential parcels, and hence the land-use-type variable is applicable only to the attraction-end models. HBO trips attracted to institutional and commercial parcels are shorter compared to similar trips attracted to any other type of parcel. The coefficient on the commercial parcel becomes statistically insignificant in the disaggregate model after explicitly controlling for the purpose of trip (shopping). Industrial parcels attract the longest $\mathrm{HBO}$ trips. A second parcel-level characteristic is the square footage of the building in the parcel. This attribute was also defined for only nonresidential parcels, and the attractionend models indicate that larger-size establishments attract longer HBO trips. Residential parcels were characterized in terms of the number of units in the parcel (single unit, 2 to 10 units, and more than 10 units). No statistically significant differences were estimated in trip lengths produced across these categories. 
Table 4: Model for HBO trip lengths.

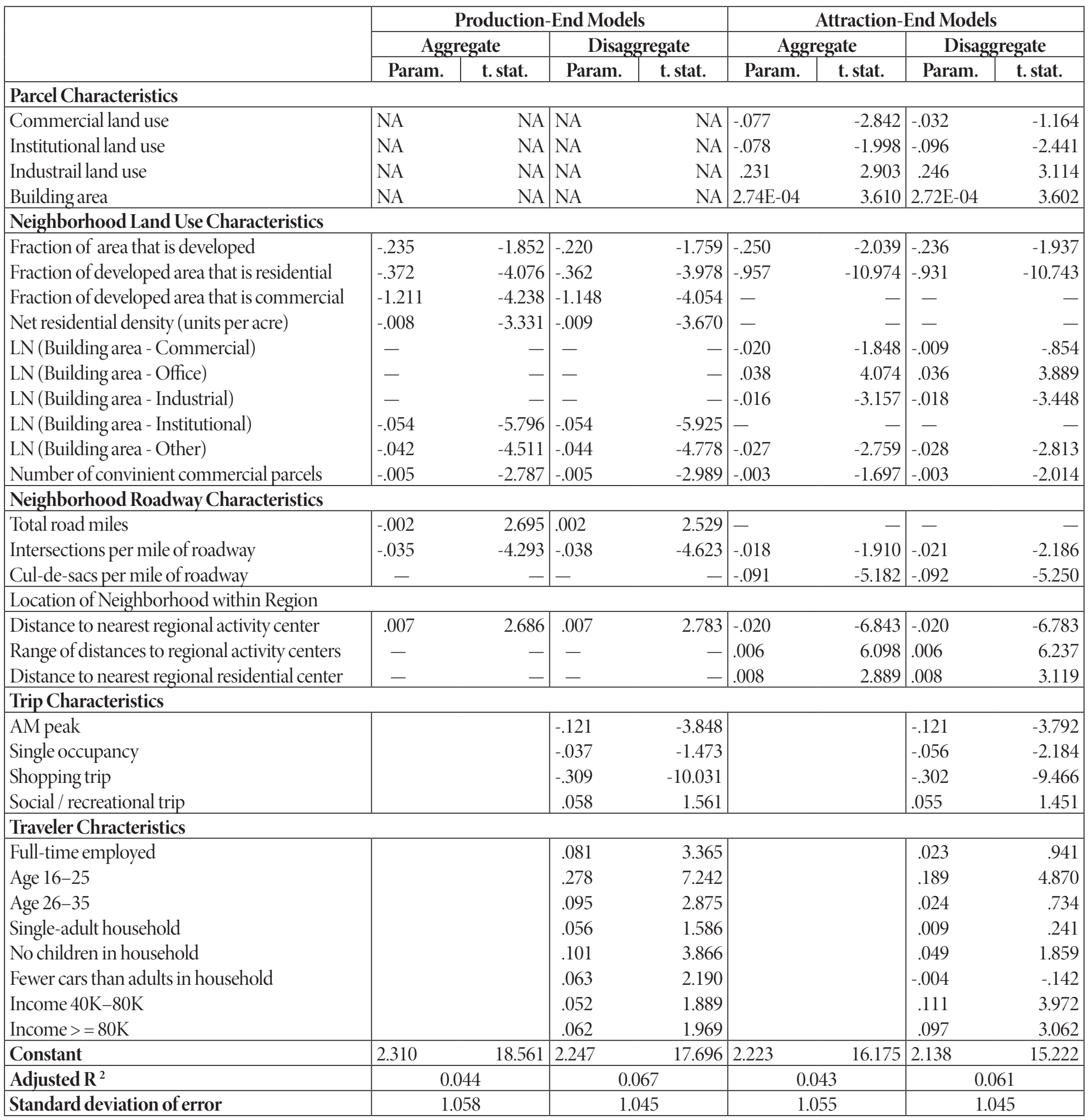


The next category of explanatory variables is neighborhood land-use characteristics. The first variable of interest is the fraction of area that is developed. In all four models, the coefficient on this variable is negative, indicating that $\mathrm{HBO}$ trips produced in and attracted to more-developed neighborhoods are shorter. The next set of variables captures the fraction of developed area by each land-use type. Unlike in the case of HBW trips, $\mathrm{HBO}$ trips produced in neighborhoods with greater residential fraction are shorter. Note that the attraction end of $\mathrm{HBO}$ trips can be a residential parcel: for example, a trip to visit a friend. At the same time, $\mathrm{HBO}$ trips attracted to areas, which are substantially residential, are also shorter (consistent with the result for HBW trips). With an increasing fraction of the neighborhood being residential, there is a greater chance that the trip is produced at a closer location. HBO trips produced in neighborhoods with a larger fraction of commercial area are shorter, indicative of higher opportunities for nonwork activities such as shopping in the vicinity of the production location, leading to shorter trips.

Residential density is negatively correlated with the length of HBO trips in the production-end models. This implies that nonwork trips produced in high-density neighborhoods are shorter.

In addition to the proportion of area under different landuse types, the size of the buildings (in square feet) is also found to be a strong predictor of trip lengths. $\mathrm{HBO}$ trips attracted to neighborhoods with large office spaces are longer. HBO trips attracted to neighborhoods with large commercial, industrial, and other floor space are shorter. (The effect of the size of commercial buildings is insignificant in the disaggregate model that controls for the shopping purpose.) The final variable characterizing the neighborhood land use is the number of parcels classified as convenient commercial (such as gas stations and drive-through establishments). The greater the number of such parcels the shorter the $\mathrm{HBO}$ trips. This variable is not statistically significant in the case of HBW trips, as gas stations and drive-through restaurants are not major employment centers.

The third category of explanatory variables is neighborhood roadway characteristics. The roadway length and intersection density (per mile) at the production end are significant predictors of trip length. Specifically, for a given length of roadway, increasing the number of intersections per mile decreases the trip length, reflective of greater connectivity leading to shorter travel distances from one point to another. At the same time, for a given intersection density, increasing the length of roadways leads to longer trips. This is perhaps reflective of coverage: a greater proportion of the neighborhood can be reached with a greater roadway length. The number of intersections per road mile and the number of cul-de-sacs per road mile at the attraction end negatively impact trip length (the latter to a greater extent than the former). While the effect of the number of intersections could be ascribed to better con- nectivity, the impact of cul-de-sacs is interesting. HBO trips attracted to neighborhoods with a large number of cul-de-sacs could be short trips to visit friends or family within a suburbanstyle neighborhood. Breaking down the nonwork trips further by purpose—such as shopping, social, or recreation-would be more illuminating.

The fourth category of explanatory variables is location of the neighborhood within the region. With increasing distance of the production end (neighborhood) of the trip to the regional activity centers, the lengths of $\mathrm{HBO}$ trips increase. Alternatively, $\mathrm{HBO}$ trips produced closer to regional activity centers are shorter, potentially because of the increased number of opportunities located near the neighborhood. The same variable has the opposite effect on the length of the HBO trips attracted. HBO trips attracted to locations near major residential centers are shorter.

The fifth and sixth categories of variables are applicable to only the disaggregate models. Among the trip characteristics, HBO trips made during the a.m. peak are the shortest. Trips made using a single-occupant vehicle are marginally shorter than those made in a car pool. Among the various types of $\mathrm{HBO}$ trips, those for shopping are the shortest and those for social/recreational purposes are marginally longer than trips for any other purpose. On examining the impacts of traveler characteristics, we find that full-time workers make longer $\mathrm{HBO}$ trips compared to part-time workers and nonworkers; perhaps these are trips chained with the commute, which is typically longer. The length of $\mathrm{HBO}$ trips decreases with an increase in the age of the traveler. Persons in single-adult households and those without children make longer $\mathrm{HBO}$ trips. $\mathrm{HBO}$ trips made by individuals who must share cars (fewer cars than adults in the household) are shorter. Interestingly, most of these factors are not significant in the attraction-end models. Finally, we see that the length of $\mathrm{HBO}$ trips increases with an increase in household income. The excluded income categories in the model are "income less than or equal to $\$ 40,000$ " and "unknown income." Thus, we find that HBW trips made by persons who did not report their income are comparable in length to those made by persons with income greater than $\$ 40,000$ (in contrast to the models for HBW trips, for which the travelers with missing income were more similar to middle-income persons). In general, the effects of trip and traveler characteristics are intuitively reasonable. Further, the land-use variables still retain their significance even after controlling for these trip and traveler characteristics.

The estimated variances of the error terms are large, or equivalently, the $\mathrm{R}^{2}$ values are small. This indicates that the included variables "explain" the variability in trip lengths only to a limited extent (between 4 percent and 7 percent in the variability in the logarithm of the trip lengths across the four models). As in the case of HBW trips, the land-use variables explain the variability to a greater extent than socioeconomics. 
The reader will note that the models were developed for an aggregate category of "nonwork trips" (the disaggregate models have partial control for trip purpose). Segmentation of this model by purpose, such as shopping, social/recreational, and personal business, can be expected to improve the model. The survey data used in this study had information on activity purpose for only the first stop of any home-based tour, with the second and later stops simply classified as "other purpose." This limited us from adopting a fully segmented approach for home-based nonwork trips.

\subsection{Models for the lengths of non-home-based (NHB) trips}

Table 5 presents the models for the lengths of non-home-based trips. The structure of this table is similar to those of Tables 3 and 4 . The rest of the discussion also follows the same structure as the previous sections on $\mathrm{HBW}$ and $\mathrm{HBO}$ trips. In the case of non-home-based trips, the production end of the trip is also its origin and the attraction end of the trip is also its destination.

The first category of explanatory variables is parcel characteristics. NHB trips produced in or attracted to commercial parcels are shorter. This is perhaps reflective of multiple shopping trips chained together with the shopping destinations close to each other. Further, larger-sized establishments produce and attract longer NHB trips.

The next category of explanatory variables is neighborhood land-use characteristics. The first variable of interest is the fraction of area that is developed. As in the case of HBW and $\mathrm{HBO}$ trips, the coefficients on this variable are negative in all of the models for NHB trips. Thus, more developed neighborhoods produce and attract shorter NHB trips. The next variable of interest is "fraction of remaining developed area." This is calculated as the proportion of developed area in all land-use types except the land-use type of the production or attraction end parcel, as appropriate. For example, for a trip produced in a commercial land parcel, the above variable determines the fraction of developed area in the neighborhood in the other five land-use types (residential, office, institutional, industrial, and other). Overall, this variable is envisioned as a measure of activity opportunities for NHB trips produced in a parcel, and in this context, the negative sign on this variable appears reasonable. The variable building area in "remaining" land-use types was defined as the building square footage in all (nonresidential) land-use types except the land-use type of the production- or attraction-end parcel as appropriate. For example, for a trip produced in a commercial land parcel, the above variable determines the floor area in the following nonresidential land-use types: office, institutional, industrial, and other. The negative sign on this variable appears reasonable and is possibly indicative of the activity opportunities in the vicinity of the production end of the NHB trip. The final variable characterizing the neighborhood land use is the number of parcels classified as convenient commercial (e.g., gas stations and drive-through establishments). The greater the number of such parcels, the shorter the NHB trips.

The impacts of neighborhood roadway characteristics at the attraction end of NHB trips are the same as the impacts of the same characteristics at the production end of these trips. Specifically, for a given length of roadway, increasing the number of intersections per mile decreases the trip length. This is perhaps reflective of greater connectivity leading to shorter travel distances to get from one point to another. At the same time, for a given intersection density, increasing the length of roadways leads to longer trips. This is perhaps reflective of coverage: a greater proportion of the neighborhood can be reached with a greater roadway length.

The fourth category of explanatory variables is location of the neighborhood within the region. NHB trips produced in locations father away from regional activity centers are shorter. A straightforward explanation of this effect is not apparent.

Few trip and traveler characteristics are found to be statistically significant predictors of the length of NHB trips. NHB trips made midday are shorter and those based at work (one end of the trip is work) are longer. Younger persons (younger than 35) are estimated to have longer trips as are those from car-sharing households.

Overall, relatively fewer explanatory factors turned out to be statistically significant in the models for non-home-based trips. Correspondingly, these models have the lowest ${ }^{\mathrm{R} 2}$ values (between 2.5 percent and 5 percent in the variability in the logarithm of the trip lengths across the four models is explained by the model). It appears intuitively reasonable that the trip-end land uses have little explanatory power in the models for nonhome-based trips. After all, unlike the spatial fixity provided by the residential location in the case of home-based trips, there are no natural spatial "pegs" in the case of non-home-based trips. These are sandwiched between other home-based trips, and the choice of trip-end locations is perhaps guided to a large extent by the spatial characteristics of the preceding and/or succeeding trips. To deal with this issue, one needs to go beyond the conventional trip-based analysis framework into modeling trip chains and tours. 
Table 5: Model for NHB trip lengths.

\begin{tabular}{|c|c|c|c|c|c|c|c|c|}
\hline & \multicolumn{4}{|c|}{ Production-End Models } & \multicolumn{4}{|c|}{ Attraction-End Models } \\
\hline & \multicolumn{2}{|c|}{ Aggregate } & \multicolumn{2}{|c|}{ Disaggregate } & \multicolumn{2}{|c|}{ Aggregate } & \multicolumn{2}{|c|}{ Disaggregate } \\
\hline & Param. & t. stat. & Param & t. stat. & Param & t. stat. & Param & t. stat. \\
\hline \multicolumn{9}{|l|}{ Parcel Characteristics } \\
\hline Commercial land use & -.099 & -2.517 & -.073 & -1.877 & -.144 & -3.686 & -.114 & -2.950 \\
\hline Building area & 5.44E-04 & 5.264 & 5.16E-04 & 5.045 & 3.37E-04 & 2.944 & 3.17E-04 & 2.803 \\
\hline \multicolumn{9}{|l|}{ Neighborhood Land Use Characteristics } \\
\hline Fraction of area that is developed & -.462 & -2.902 & -.364 & -2.307 & -.346 & -2.186 & -.273 & -1.740 \\
\hline Fraction of developed area in remaining land uses & -.295 & -4.513 & -.278 & -4.306 & -.170 & -2.550 & -.163 & -2.475 \\
\hline LN (Building area - Remaining) & -.052 & -2.899 & -.057 & -3.215 & -.049 & -2.847 & -.055 & -3.220 \\
\hline Number of convinient commercial parcels & -.008 & -4.187 & -.008 & -4.047 & -.009 & -4.299 & -.009 & -4.427 \\
\hline \multicolumn{9}{|l|}{ Neighborhood Roadway Characteristics } \\
\hline Total road miles & .004 & 4.428 & .004 & 4.046 & .004 & 3.731 & .003 & 3.413 \\
\hline Intersections per mile of roadway & -.020 & -1.675 & -.022 & -1.872 & -.035 & -2.828 & -.035 & -2.876 \\
\hline \multicolumn{9}{|l|}{ Location of Neighborhood within Region } \\
\hline Distance to nearest regional activity center & -.006 & -1.778 & -.006 & -1.706 & - & - & - & - \\
\hline \multicolumn{9}{|l|}{ Trip Characteristics } \\
\hline Mid day & & & -.237 & -7.268 & & & -.240 & -7.381 \\
\hline Work based trip & & & .233 & 7.056 & & & .246 & 7.438 \\
\hline \multicolumn{9}{|l|}{ Traveler Characteristics } \\
\hline Age 16-25 & & & .095 & 1.527 & & & .091 & 1.464 \\
\hline Age 26-35 & & & .107 & 2.495 & & & .104 & 2.424 \\
\hline Fewer cars than adults in household & & & .062 & 1.547 & & & .061 & 1.544 \\
\hline Constant & 2.152 & 12.419 & 2.143 & 12.422 & 2.133 & 14.703 & 2.129 & 14.726 \\
\hline Adjusted $\mathrm{R}^{2}$ & \multirow{2}{*}{\multicolumn{2}{|c|}{$\begin{array}{l}0.025 \\
1.131\end{array}$}} & \multirow{2}{*}{\multicolumn{2}{|c|}{$\begin{array}{l}0.049 \\
1.117\end{array}$}} & \multirow{2}{*}{\multicolumn{2}{|c|}{$\begin{array}{l}0.024 \\
1.132\end{array}$}} & \multirow{2}{*}{\multicolumn{2}{|c|}{$\begin{array}{l}0.049 \\
1.117 \\
\end{array}$}} \\
\hline Standard deviation error & & & & & & & & \\
\hline
\end{tabular}

\section{$4 \quad$ Application}

As an illustration of model application, the aggregate production-end models estimated are used to predict the lengths of HBW and HBO trips produced in identical residential parcels located in three very different neighborhoods of the region: one in Pahokee, in rural Palm Beach County, the second just outside the city of Palm Beach (suburban), and the third in downtown Miami (Figure 2). Similarly, the aggregate attraction-end models are used to predict the lengths of $\mathrm{HBW}$ and $\mathrm{HBO}$ trips attracted to a commercial parcel with a 50,000-square-foot building (a large supermarket) when located in the same three neighborhoods. Note that the aggregate models are used here because the socioeconomics of the travelers are generally unknown in transportation-impact studies.

The models were implemented in a spreadsheet program (Figure 3), and this was used to generate all results presented in this application section. The residential parcel in the rural neighborhood produces the longest HBW (19.61 miles) and HBO trips (8.68 miles). The apparent lack of employment opportunities and the relatively long distances to the activity centers along the coast make this estimation reasonable. The parcel in the urban location produces the shortest HBW (4.21) and HBO (2.23) trips. The suburban location in West Palm Beach produced HBW trips of 6.72 miles and $\mathrm{HBO}$ trips of 3.85 miles. In the case of the commercial development, the supermarket, when located in the rural location, attracted the shortest trips ( 4.9 for $\mathrm{HBW}$ and 4.2 for $\mathrm{HBO}$ ), whereas the same store located in urban or suburban regions attracted longer trips (approximately 11 miles for HBW and 8 miles for HBO). In rural and suburban location, there are a lot more residences in the vicinity of the new commercial development from which to attract trips. 

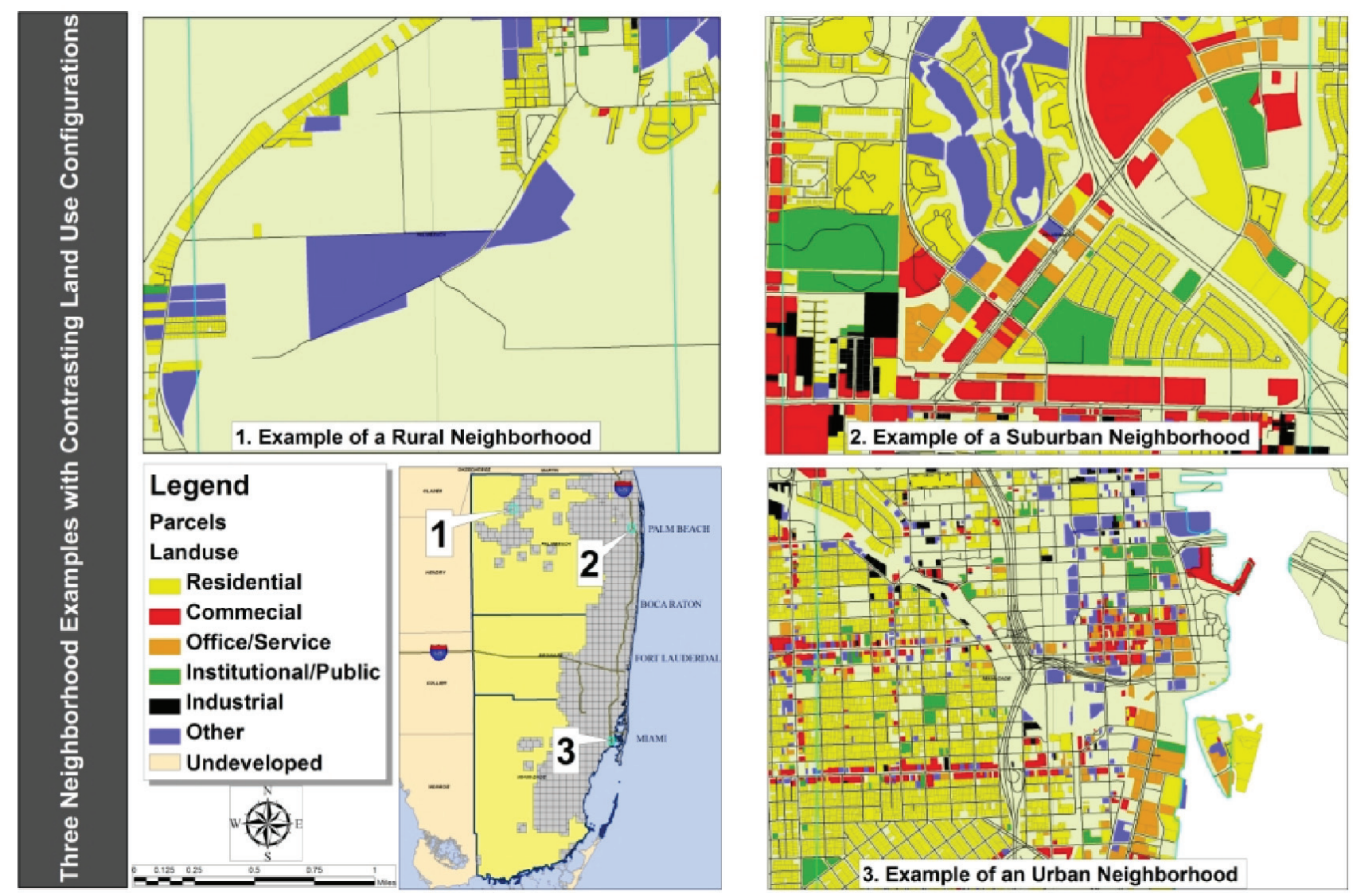

Figure 2: Location and characteristics of three neighborhoods for model application.

Overall, this example illustrates the ability of the models to predict trip lengths reflective of the context in which the travel is taking place. Further, if the socioeconomics of the travelers are known, the disaggregate models can be applied to predict the expected trip lengths for each of the population segments. At the same time, it is useful to note that the spreadsheet calculates point estimates of trip lengths. As the logarithm of the trip length is modeled using the linear regression structure, one can also calculate the variance associated with this point estimate using the properties of the log-normal distribution. In the examples discussed above, the estimated standard deviation of the prediction trip length is approximately equal to the mean, indicating that the predictions are not very robust. This is as expected given that the standard deviations of the error terms in the models were estimated to be large (see the last row in tables 3, 4, and 5). As discussed previously, the robustness of the prediction can be improved using additional explanatory variables, among other things. However, such an approach will also require additional data for model application. 


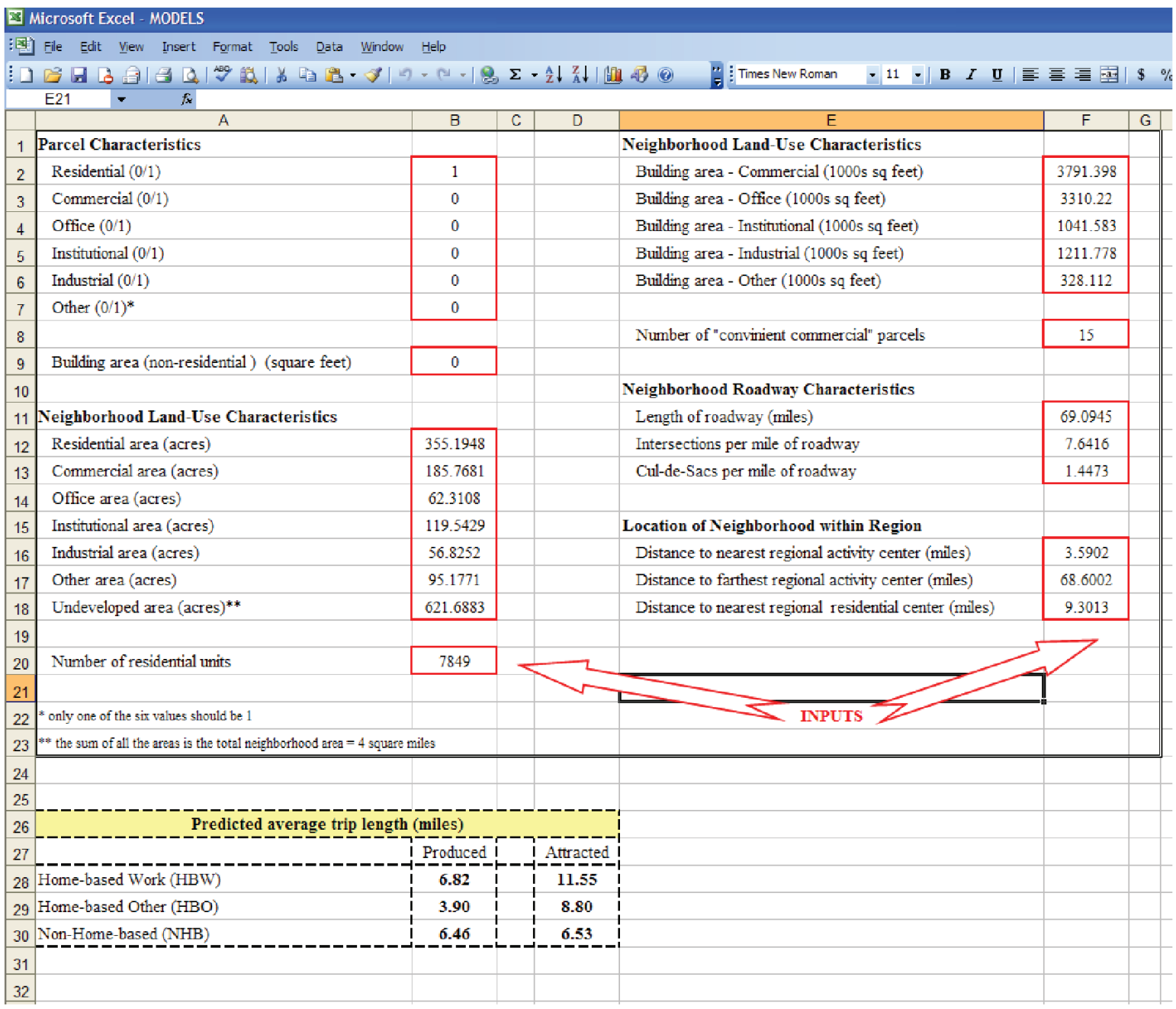

Figure 3: Spreadsheet implementation of the trip-length models. 


\section{$5 \quad$ Summary and conclusions}

This study developed models that relate trip lengths to the land-use characteristics at the trip ends (both production and attraction ends). Separate models were developed by trip purpose. The results indicate several statistically significant and intuitively reasonable effects of land-use patterns. High residential densities and a good mix of complementary land uses are associated with shorter trips. Larger establishments attract longer trips, and the $\mathrm{HBO}$ trip lengths decrease with an increased number of convenient-commercial land-use parcels in the neighborhood. The connectivity provided by the roadway network (measured in terms of number of intersections and cul-de-sacs) affects trip lengths. In addition to the local (neighborhood-level) land-use characteristics, trip lengths also significantly vary by the location of the neighborhood within the region. All these results hold even after controlling for several trip and traveler characteristics. Incorporating traveler attitudes and lifestyles is a potential empirical extension to the current work. Further, evaluating the stability of the impact of land-use variables under alternate definitions of neighborhoods (circular instead of square, different sizes, etc.) would also be very beneficial.

The models have been implemented in a spreadsheet program for use in transportation-impact analysis. We believe that this spreadsheet is a valuable tool, especially for local governments that might lack an elaborate model for transportationdemand forecasting. Readers will also note that our (aggregate) models rely only on the land-use patterns around the land parcel being developed; data that can be assembled with reasonable ease. A key guiding principle of our approach was to use descriptors of land use (such as proportions of land uses by type) and transportation system characteristics (roadway lengths and number of intersections and cul-de-sacs) that can be constructed fairly easily in an application context. While it is very reasonable to expect that variables that describe the network topology (such as "tree density") and land-use (such as dissimilarity and adjacency of complementary land-use types) are strongly correlated to trip lengths, these would require additional (GIS) effort that might not be available in the application context.

Overall, we believe that our models represent a significant effort to analyze trip lengths associated with land developments within the trip-based travel-forecasting paradigm and using explanatory measures that can be easily constructed in the application context. At the same time, there are clear avenues for improving the models both empirically (use of additional explanatory variables and development of models for disaggregate trip purposes) and methodologically (incorporating trip chains and use of spatially correlated models) to produce more robust (lower variance) trip-length predictions. However, the application of such models in practice would require additional data, GIS-skills, and the adoption of tour-based/activity-based modeling paradigms.

We envision that the empirical insights from this study could inform future developments of activity-based, traveldemand model systems to comprehensively address all aspects of changes in travel behavior because of land developments. Such models could be used to evaluate the impacts of alternate land-development patterns and thus help in the design of urban areas that have shorter trip lengths, less fuel consumption, and lower GHG emissions.

\section{References}

Bagley, M. N., and P. L. Mokhtarian. 2002. The impact of residential neighborhood type on travel behavior: a structural equations modeling approach. The Annals of Regional Science, 36(2): 279-297.

Bento, A. M., M. L. Cropper, A. M. Mobarak, and K. Vinha. 2005. The effects of urban spatial structure on travel demand in the United States. The Review of Economics and Statistics, 87(3): 466-478.

Brownstone, D., and T. F. Golob. 2009. The impact of residential density on vehicle usage and energy consumption. Journal of Urban Economics, 65: 91-98.

Cervero, R., and M. Duncan. 2006. Which reduces travel more: jobs-housing balance or retail-housing mix? Journal of the American Planning Association, 72(4): pp.475-492.

Cervero, R., and K. M. Kockelman. 1997. Travel demand and the 3Ds: Density, diversity, and design. Transportation Research Part D, 2(3): 199-219.

Handy, S., X. Cao, and P. Mokhtarian. 2005. Correlation or causality between the built environment and travel behavior? Evidence from Northern California. Transportation Research Part D: Transport and Environment, 10(6): 427-444.

Holtzclaw, J., R. Clear, H. Dittmar, D. Goldstein, and P. Haas. 2002. Location efficiency: Neighborhood and socioeconomic characteristics determine auto ownership and usestudies of Chicago, Los Angeles, and San Francisco. Transportation Planning and Technology, 25(1): 1-27.

Krizek, K. J. 2003. Residential relocation and changes in urban travel: does neighborhood-scale urban form matter? Journal of the American Planning Association, 69(3): 265-279.

Ewing, R., and R. Cevero. 2001. Travel and the built environment: A synthesis. Transportation Research Board, 1780(1): 87-114.

Ewing, R., P. Haliyur, and G. Page. 1994. Getting around a traditional city, suburban planned unit development and everything in between. Transportation Research Record, 1466: 53-62.

Frank, L., J. Chapman, M. Bradley, and K. Lawton. 2005. Travel behavior emissions and land use correlation analy- 
sis in the Puget Sound. Washington State Department of Transportation. http://www.wsdot.wa.gov/research/ reports/fullreports/625.1.pdf.

Handy, S. L. 1996. Understanding the link between urban form and nonwork travel behavior. Journal of Planning Education and Research, 15(3): 183-198.

Parthasarathi, P., H. Hochmair, and D. Levinson. 2011. Network structure and spatial separation. 90th Transportation Research Board Annual Meeting, Washington, DC.

Parthasarathi, P. and D. Levinson. 2011. Network structure and metropolitan mobility. 90th Transportation Research Board Annual Meeting, Washington, DC.

Rutherford, G. S., E. McCormack, and M. Wilkinson. 1996. Travel impacts of urban form: Implications from an analysis of two Seattle area travel diaries. TMIP Conference on Urban Design, Telecommuting, and Travel Behavior, October 27-30. Williamsburg, VA.

Transportation Research Board. (2009). Driving and the built environment: The effects of compact development on motorized travel, energy use, and CO2 emissions. http://onlinepubs.trb. org/onlinepubs/sr/sr298prepub.pdf (Accessed June 2011). 